\title{
Construção eValidação de um Protocolo de Cuidados para Pacientes Críticos com Câncer em Delirium
}

doi: https://doi.org/10.32635/2176-9745.RBC.2021v67n3.1311

Construction and Validation of a Care Protocol for Critical Patients with Cancer in Delirium
Construcción y Validación de un Protocolo de Atención al Paciente Crítico con Cáncer en Delirium

Bárbara Rocha Gouveia'; Rafael Tavares Jomar²; Débora Cristina Leitão dos Santos³; Tânia Cristina de Oliveira Valente ${ }^{4}$

RESUMO

Introduçáo: A frequência de delirium em unidades de terapia intensiva oncológica é elevada. Objetivo: Construir um protocolo de cuidados para pacientes com câncer em delirium, internados em uma unidade de terapia intensiva e investigar sua validade de face e de conteúdo. Método: Estudo descritivo realizado em 2016 em um centro de assistência de alta complexidade em oncologia localizado na cidade do Rio de Janeiro, Brasil. Um comitê formado por 43 juízes apreciou as validades de face e de conteúdo do protocolo que foi construído a partir das recomendaçóes constantes no Clinical Practice Guidelines for the Management of Pain, Agitation, and Delirium. Calcularam-se o índice de validade de conteúdo e as proporçôes de pertinência e de conformidade de cada item desse protocolo. Resultados: Todos os 19 itens obtiveram índice de validade de conteúdo >0,80 e proporçóes de conformidade superiores a 95\%. A proporção de pertinência de cada item variou de $86 \%$ a $100 \%$. Conclusáo: O protocolo apresentou adequadas validades de face e de conteúdo, mostrando-se promissor no manejo do delirium em pacientes com câncer internados em unidade de terapia intensiva.

Palavras-chave: Delírio; Unidades de Terapia Intensiva; Institutos de Câncer; Estudos de Validação.

\section{ABSTRACT}

Introduction: The frequency of delirium in oncology intensive care units is high. Objective: To build a care protocol for cancer patients with delirium admitted to an intensive care unit and investigate its face and content validity. Method: Descriptive study conducted in 2016 in a high complexity care center in oncology located in the city of Rio de Janeiro, Brazil. A committee formed by 43 judges assessed the face and content validity of the protocol that was built from the recommendations contained in the Clinical Practice Guidelines for the Management of Pain, Agitation, and Delirium. The content validity index and the proportions of pertinence and compliance of each item of the protocol were calculated. Results: All 19 items had a content validity index $>0.80$ and proportions of compliance greater than $95 \%$. The relevance ratio of each item ranged from $86 \%$ to $100 \%$. Conclusion: The protocol presented adequate face and content validity, being promising for the management of delirium in cancer patients admitted to an intensive care unit.

Key words: Delirium; Intensive Care Units; Cancer Care Facilities; Validation Study.

\section{RESUMEN}

Introducción: La frecuencia del delirium en las unidades de cuidados intensivos oncológicos es alta. Objetivo: Elaborar un protocolo de atención para pacientes oncológicos con delirium ingresados en unidad de cuidados intensivos e investigar su validez aparente y de contenido. Método: Estudio descriptivo realizado en 2016 en un centro de atención de alta complejidad en oncología ubicado en la ciudad de Río de Janeiro, Brasil. Un comité formado por 43 jueces evaluó la validez aparente y de contenido del protocolo que se construyó a partir de las recomendaciones contenidas em el Clinical Practice Guidelines for the Management of Pain, Agitation, and Delirium. Se calculó el índice de validez de contenido y las proporciones de pertinencia y cumplimiento de cada ítem del protocolo. Resultados: Los 19 ítems tenían un índice de validez de contenido $>0,80$ y proporciones de cumplimiento superiores al $95 \%$. El índice de relevancia de cada ítem osciló entre el $86 \%$ y el $100 \%$. Conclusión: El protocolo presentó una adecuada validez aparente y de contenido, y se mostró prometedor en el manejo del delirium en pacientes con cáncer ingresados en una unidad de cuidados intensivos.

Palabras clave: Delirio; Unidades de Cuidados Intensivos; Instituciones Oncológicas; Estudio de Validación.

1-3/nstituto Nacional de Câncer José Alencar Gomes da Silva (INCA). Rio de Janeiro (RJ), Brasil.

${ }^{4}$ Departamento de Saúde Coletiva do Instituto Biomédico da Universidade Federal do Estado do Rio de Janeiro. Rio de Janeiro (RJ), Brasil.

1E-mail: barbarargouveia@gmail.com. Orcid iD: https://orcid.org/0000-0002-2618-5758

${ }^{2}$ E-mail: rafaeljomar@yahoo.com.br. Orcid iD: https://orcid.org/0000-0002-4101-7138

${ }^{3}$ E-mail: dcleitao81@gmail.com. Orcid iD: https://orcid.org/0000-0003-1475-7541

${ }^{4}$ E-mail: valenteunirio@gmail.com. Orcid iD: https://orcid.org/0000-0002-5735-5983

Endereço para correspondência: Bárbara Rocha Gouveia. Praça Cruz Vermelha, 23 - Centro. Rio de Janeiro (RJ), Brasil. CEP 20230-130. E-mail: barbarargouveia@gmail.com 


\section{INTRODUÇÃO}

$\mathrm{O}$ delirium corresponde a uma disfunção neurológica aguda caracterizada por alteraçóes transitórias da consciência e da cognição observada com frequência em pacientes internados em unidades de terapia intensiva (UTI). A prevalência desse fenômeno é de cerca de $70 \%$ e a incidência pode chegar a até $89 \%$ em UTI não especializadas ${ }^{1-3}$. Em UTI oncológicas, a prevalência e a incidência de delirium também são elevadas, especialmente entre pacientes sob ventilação mecânica, respectivamente, $95 \%{ }^{4}$ e $64,6 \%{ }^{5}$.

Além do mais, pacientes que desenvolvem delirium costumam apresentar piores resultados na evolução, o que contribui para o aumento do tempo de hospitalização, maior risco de ocorrência de eventos adversos, a exemplo de complicações respiratórias e neurológicas, além de maior mortalidade ${ }^{1-3}$.

A fim de contribuir para a implementaçáo de estratégias de avaliação, prevençáo e tratamento do delirium em ambientes críticos, o objetivo deste estudo foi construir um protocolo de cuidados para pacientes com câncer em delirium internados em UTI e investigar sua validade de face e de conteúdo.

\section{MÉTODO}

Estudo descritivo de caráter metodológico que foi conduzido entre os meses de outubro e dezembro de 2016 na UTI de um centro de assistência de alta complexidade em oncologia localizado na cidade do Rio de Janeiro, Brasil.

Para construção do protocolo de cuidados, utilizaram-se as recomendaçôes constantes no Clinical Practice Guidelines for the Management of Pain, Agitation, and Delirium $^{6}$, do qual foram selecionados itens considerados relevantes, sendo tal relevância confirmada por extensa revisão de literatura realizada pelos autores. Os 19 itens selecionados foram distribuídos em dimensões nomeadas "Dor e Analgesia", "Agitação e Sedação" e "Estratégias de Manejo”.

A técnica Delphi ${ }^{7}$ foi aplicada para validação de face e de conteúdo do protocolo de cuidados por um comitê de juízes formado por enfermeiros, médicos e fisioterapeutas. Os critérios para a seleção de juízes foram tempo de experiência prática em terapia intensiva maior ou igual a cinco anos e tempo de atuação no cenário de estudo maior ou igual a um ano.

Assim, 47 juízes receberam carta-convite, Termo de Consentimento Livre e Esclarecido (TCLE) e link para acessar a primeira versáo do protocolo de cuidados por e-mail, por $a p p$ de troca de mensagens ou das máos da autora principal do estudo. Nessa etapa, os juízes foram orientados a avaliar a aparência e o conteúdo do protocolo de cuidados mediante análise dos itens selecionados para sua composição por meio de um questionário com espaço para comentários e sugestôes. Sobre a pertinência de cada item no protocolo, os juízes deveriam assinalar umas das seguintes opçôes do questionário: "concordo" ou "discordo". No tocante ao grau de importância de cada item, os juízes deveriam assinalar uma das seguintes opçôes do questionário: "sem importância", "importante" ou "muito importante".

Devolveram o TCLE assinado e o questionário devidamente preenchido no prazo estabelecido (até 20 dias) 43 juízes. Em seguida, calculou-se o índice de validade de conteúdo (IVC), que mediu a proporção de juízes que estavam em concordância sobre o grau de importância de cada item classificado como "importante" ou "muito importante", sendo considerados válidos aqueles que obtiveram valor mínimo de 0,80 de concordância entre os juízes 8 .

Comentários e sugestôes dos juízes feitos na etapa anterior foram considerados na seguinte, na qual a redação de alguns itens foi reformulada e um novo item foi incluído no protocolo de cuidados. Na sequência, a versão reformulada do protocolo foi submetida a novo exame dos mesmos 43 juízes, que devolveram o questionário de avaliação em, no máximo, dez dias após seu recebimento por e-mail, por app de troca de mensagens ou das máos da autora principal do estudo. Nesse questionário, os juízes responderam "sim" ou "não" sobre a conformidade dos itens componentes do protocolo no tocante à clareza e à forma como os itens estavam agrupados, à coerência entre os itens apresentados e suas respostas, à objetividade dos itens e à facilidade de leitura e compreensão, segundo proposta de avaliação de conformidade sugerida por Pasquali'.

A conduçấo do estudo foi aprovada pelos Comitês de Ética em Pesquisa da Universidade Federal do Estado do Rio de Janeiro (Parecer 1.769.591) e do Instituto Nacional de Câncer José Alencar Gomes da Silva (Parecer 1.776.393) em outubro de 2016.

\section{RESULTADOS}

O comitê foi composto 22 enfermeiros, 16 médicos e cinco fisioterapeutas, majoritariamente, do sexo feminino (67,4\%). A média de idade dos juízes foi igual a 40,7 anos (desvio-padrão: 7,0) e o tempo médio de experiência prática em terapia intensiva igual a 14,4 anos (desvio-padrão: 2,0). Todos possuíam curso de pós-graduação na área de terapia intensiva, sendo que $27,9 \%$ eram mestres e $7,0 \%$ doutores. 
A primeira versão do protocolo de cuidados avaliada pelo comitê está apresentada no Quadro 1.

Sobre a pertinência de cada item no protocolo, a proporção de concordância dos juízes variou de $86 \%$ a $100 \%$, sendo que 14 dos 19 itens obtiveram proporção de concordância $\geq 95 \%$. Conforme a Tabela 1 , todos os itens

Quadro 1. Primeira versão do protocolo

\begin{tabular}{|c|c|}
\hline \multirow{3}{*}{ 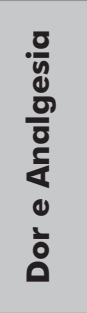 } & $\begin{array}{l}\text { 1. Incidência de dor (considerar dor } \\
\text { oncológica) }\end{array}$ \\
\hline & $\begin{array}{l}\text { 2. Avaliação da dor (utilizar, } \\
\text { preferencialmente, a Behavioral Pain Scale } \\
\text { para pacientes sedados ou a escala visual } \\
\text { analógica para os não sedados) }\end{array}$ \\
\hline & 3. Tratamento/Controle da dor \\
\hline \multirow{4}{*}{ 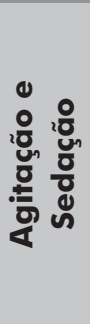 } & 4. Despertar diário programado e assistido \\
\hline & $\begin{array}{l}\text { 5. Evitar uso abusivo de medicamentos } \\
\text { sedativos em bolus }\end{array}$ \\
\hline & $\begin{array}{l}\text { 6. Monitoramento da profundidade da } \\
\text { sedação (utilizar a Richmond Agitation- } \\
\text {-Sedation Scale }{ }^{11} \text { ) }\end{array}$ \\
\hline & 7. Escolha do sedativo \\
\hline \multirow{12}{*}{ 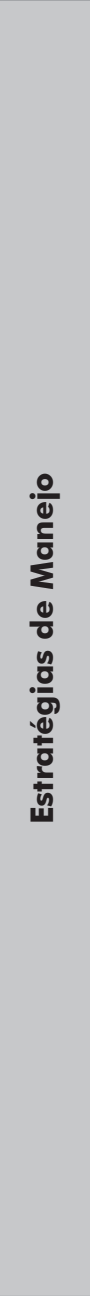 } & $\begin{array}{l}\text { 8. Detecção e monitoramento de delirium } \\
\text { (utilizar o Confusion Assessment Method for } \\
\text { the Intensive Care Unit }{ }^{12} \text { ) }\end{array}$ \\
\hline & $\begin{array}{l}\text { 9. Sempre que possível, realizar } \\
\text { mobilização precoce }\end{array}$ \\
\hline & $\begin{array}{l}\text { 10. Manter temperatura adequada de } \\
\text { acordo com condição do indivíduo }\end{array}$ \\
\hline & $\begin{array}{l}\text { 11. Evitar uso de ataduras para restrição; se } \\
\text { necessário, privilegiar luva de boxe }\end{array}$ \\
\hline & $\begin{array}{l}\text { 12. Permitir a presença de acompanhante } \\
\text { sempre que possível }\end{array}$ \\
\hline & $\begin{array}{l}\text { 13. Propiciar visita à unidade pós- } \\
\text {-operatória em casos de cirurgia eletiva }\end{array}$ \\
\hline & $\begin{array}{l}\text { 14. Utilizar protetores auriculares avaliando } \\
\text { a condição individual }\end{array}$ \\
\hline & $\begin{array}{l}\text { 15. Manter luz indireta apenas e instalar } \\
\text { dispositivos de controle de luminosidade }\end{array}$ \\
\hline & $\begin{array}{l}\text { 16. Manter próteses auditivas e permitir } \\
\text { uso dos óculos e de outros objetos pessoais } \\
\text { sempre que possível }\end{array}$ \\
\hline & $\begin{array}{l}\text { 17. Evitar uso de aparelhos de televisão e } \\
\text { rádio após as } 24: 00 \text { horas (utilizar, quando } \\
\text { indicado, fones de ouvido) }\end{array}$ \\
\hline & $\begin{array}{l}\text { 18. Manter cortinas abertas durante o dia, } \\
\text { sempre que possível, permitindo que o } \\
\text { paciente diferencie dia/noite }\end{array}$ \\
\hline & $\begin{array}{l}\text { 19. Utilização de relógios de parede } \\
\text { digitais, permitindo que o paciente } \\
\text { diferencie horas do dia e da noite }\end{array}$ \\
\hline
\end{tabular}

da primeira versão do protocolo de cuidados obtiveram IVC $>0,80$.

Comentários e sugestóes dos juízes sobre a aparência e o conteúdo do protocolo de cuidados determinaram a

Tabela 1. Índice de validade de conteúdo de cada item da primeira versão do protocolo

Item
1. Incidência de dor (considerar dor
oncológica)
2. Avaliação da dor (utilizar,
preferencialmente, a Behavioral Pain
Scale ${ }^{10}$ para pacientes sedados ou a
escala visual analógica para os não
sedados)

IVC

3. Tratamento/Controle da dor

4. Despertar diário programado e assistido

5. Evitar uso abusivo de medicamentos sedativos em bolus

6. Monitoramento da profundidade da sedação (utilizar a Richmond Agitation-Sedation Scale ${ }^{11}$ )

7. Escolha do sedativo

8. Detecção e monitoramento de delirium (utilizar o Confusion Assessment Method for the Intensive Care Unit' ${ }^{12}$ )

9. Sempre que possível, realizar mobilização precoce

10. Manter temperatura adequada de acordo com condição do indivíduo

11. Evitar uso de ataduras para restrição; se necessário, privilegiar luva de boxe

12. Permitir a presença de acompanhante sempre que possível

13. Propiciar visita à unidade pós-operatória em casos de cirurgia eletiva

14. Utilizar protetores auriculares avaliando a condição individual 15. Manter luz indireta apenas e instalar dispositivos de controle de luminosidade 16. Manter próteses auditivas e permitir uso dos óculos e de outros objetos pessoais sempre que possível

17. Evitar uso de aparelhos de televisão e rádio após as 24:00 horas (utilizar, quando indicado, fones de ouvido) 18. Manter cortinas abertas durante o dia, sempre que possível, permitindo que o paciente diferencie dia/noite 19. Utilização de relógios de parede digitais, permitindo que o paciente diferencie horas do dia e da noite
1,00 
reformulação da redação de alguns itens, tal como o 14 e o 17, e até mesmo a combinação deles: caso do item 18 que, embora tenha obtido IVC igual a 1,00 , foi combinado ao item 15, que obteve IVC igual a 0,95 (Tabela 1). Além disso, foi incluído um item novo sobre conforto (item 19), uma vez que $11,6 \%$ dos juízes recomendaram sua inclusão na versão final do protocolo de cuidados, apresentado no Quadro 2.

Quadro 2. Versão final do protocolo

\begin{tabular}{|c|c|}
\hline \multirow{3}{*}{ 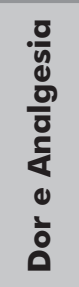 } & $\begin{array}{l}\text { 1. Avaliar a incidência de dor (considerar dor } \\
\text { oncológica) }\end{array}$ \\
\hline & $\begin{array}{l}\text { 2. Avaliar a dor (utilizar, preferencialmente, } \\
\text { a Behavioral Pain Scale }{ }^{10} \text { para pacientes } \\
\text { sedados ou a escala visual analógica para os } \\
\text { não sedados) }\end{array}$ \\
\hline & 3. Tratar a dor \\
\hline \multirow{4}{*}{ 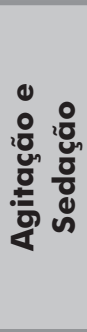 } & $\begin{array}{l}\text { 4. Programar o despertar diário programado } \\
\text { e assistido visando sedação guiada por } \\
\text { metas }\end{array}$ \\
\hline & $\begin{array}{l}\text { 5. Evitar o uso abusivo de medicamentos } \\
\text { sedativos em bolus }\end{array}$ \\
\hline & $\begin{array}{l}\text { 6. Monitorar a profundidade da sedação } \\
\text { (utilizar a Richmond Agitation-Sedation Scale }{ }^{11} \text { ) }\end{array}$ \\
\hline & 7. Escolher adequadamente o sedativo \\
\hline \multirow{12}{*}{ 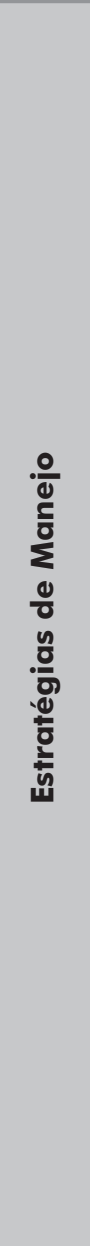 } & $\begin{array}{l}\text { 8. Detectar e monitorar o delirium (utilizar } \\
\text { o Confusion Assessment Method for the } \\
\text { Intensive Care Unit }{ }^{12} \text { ) }\end{array}$ \\
\hline & $\begin{array}{l}\text { 9. Realizar a mobilização precoce, tão logo } \\
\text { seja possível }\end{array}$ \\
\hline & $\begin{array}{l}\text { 10. Manter a temperatura corporal adequada } \\
\text { de acordo com a condição do paciente }\end{array}$ \\
\hline & $\begin{array}{l}\text { 11. Evitar o uso de ataduras para contenção } \\
\text { mecânica; se necessário, privilegiar a do tipo } \\
\text { luva boxe }\end{array}$ \\
\hline & $\begin{array}{l}\text { 12. Permitir a presença de acompanhante } \\
\text { sempre que possível }\end{array}$ \\
\hline & $\begin{array}{l}\text { 13. Propiciar uma visita prévia para } \\
\text { conhecimento da unidade pós-operatória em } \\
\text { caso de cirurgia eletiva }\end{array}$ \\
\hline & $\begin{array}{l}\text { 14. Minimizar e gerenciar os ruídos do } \\
\text { ambiente }\end{array}$ \\
\hline & $\begin{array}{l}\text { 15. Permitir a entrada de luz natural } \\
\text { para que o paciente diferencie dia e } \\
\text { noite, respeitando o período noturno com } \\
\text { luminosidade reduzida sempre que possível }\end{array}$ \\
\hline & $\begin{array}{l}\text { 16. Manter as próteses auditivas e permitir } \\
\text { o uso de óculos e de outros objetos pessoais } \\
\text { sempre que possível }\end{array}$ \\
\hline & $\begin{array}{l}\text { 17. Evitar o uso de aparelhos de televisão e } \\
\text { rádio após as } 22: 00 \text { horas (utilizar, quando } \\
\text { indicado, fones de ouvido) }\end{array}$ \\
\hline & $\begin{array}{l}\text { 18. Utilizar relógio digital de parede, } \\
\text { permitindo que o paciente diferencie as } \\
\text { horas do dia e da noite }\end{array}$ \\
\hline & 19. Proporcionar conforto \\
\hline
\end{tabular}

De acordo com a proposta de avaliaçáo de conformidade sugerida por Pasquali ${ }^{9}$, todos os itens componentes da versão final do protocolo de cuidados apresentaram proporçôes de conformidade superiores a 95\% (Tabela 2).

Tabela 2. Conformidade dos itens da versão final do protocolo

\begin{tabular}{|c|c|}
\hline Conformidade & $\operatorname{Sim}(\%)$ \\
\hline $\begin{array}{l}\text { Os itens apresentados estão em } \\
\text { conformidade com o critério de } \\
\text { objetividade? - devem permitir uma } \\
\text { resposta certa ou errada }\end{array}$ & 100 \\
\hline $\begin{array}{l}\text { Os itens apresentados estão em } \\
\text { conformidade com o critério de } \\
\text { simplicidade? - devem expressar uma } \\
\text { única ideia }\end{array}$ & 98 \\
\hline $\begin{array}{l}\text { Os itens apresentados estão em } \\
\text { conformidade com o critério de clareza? } \\
\text { - devem ser compreensíveis }\end{array}$ & 98 \\
\hline $\begin{array}{l}\text { Os itens apresentados estão em } \\
\text { conformidade com o critério de } \\
\text { variedade? - padronização de termos, } \\
\text { ocasionando uma leitura confusa, } \\
\text { cansativa e monótona }\end{array}$ & 98 \\
\hline $\begin{array}{l}\text { Os itens apresentados estão em } \\
\text { conformidade com o critério de } \\
\text { tipicidade? - presença de frases com } \\
\text { expressões condizentes à temática }\end{array}$ & 100 \\
\hline $\begin{array}{l}\text { Os itens apresentados estão em } \\
\text { conformidade com o critério de } \\
\text { amplitude? - conjunto dos itens deve } \\
\text { cobrir toda a extensão de magnitude da } \\
\text { temática }\end{array}$ & 96 \\
\hline
\end{tabular}

\section{DISCUSSÃO}

Os resultados do estudo indicam que a versão final do protocolo de cuidados para pacientes com câncer em delirium internados em UTI é composto por itens pertinentes e importantes, que foram agrupados de forma clara e coerentemente apresentados no que concerne à objetividade e à facilidade de leitura e compreensão. Diante desses resultados, acredita-se que o protocolo tenha potencial para colaborar com o manejo do delirium em pacientes críticos com câncer.

Uma revisão sistemática ${ }^{13}$ concluiu que programas de implementação de estratégias direcionadas à avaliação, à prevençấo e ao tratamento do delirium em UTI, integradas a açôes de manejo da dor e da agitação ${ }^{6}$, tal como o protocolo aqui investigado, têm potencial para melhorar os resultados clínicos dos pacientes.

O primeiro item do protocolo de cuidados trata da avaliação da dor, incluindo a dor oncológica. Esse destaque foi dado porque a dor oncológica é caracterizada por sensaçôes simultâneas de dor aguda e crônica associadas 
à disseminação de células tumorais no corpo, ou por ser consequência de uma modalidade de tratamento do câncer, ou por ter sido provocada por outras condiçóes relacionadas à doença, como ferida tumoral, por exemplo $^{14}$. Sendo assim, é imprescindível que profissionais atuantes em UTI oncológica considerem a avaliação da dor porque, além de ela ser um fator desencadeante de delirium, quando negligenciada ${ }^{15}$, é descrita como sensação intensa e insuportável acompanhada por dificuldade para dormir e irritabilidade ${ }^{14}$.

Além de o protocolo ter considerado a avaliação da dor oncológica, os juízes sugeriram a inclusão de outro item que contemplasse o conforto do paciente. O conforto já havia sido apontado por estudo anterior como importante questão a ser observada no manejo do delirium em UTI. Sabe-se que o conforto é influenciado pelos contextos físico, psicoespiritual, social e ambiental, que, notadamente, sofre influência das condiçóes de luminosidade, som e temperatura ${ }^{16}$, que tem características particulares em UTI: ruídos permanentes (oriundos de monitores cardíacos, ventiladores mecânicos e bombas infusoras de medicamentos), odores desagradáveis, iluminação artificial intensa e temperatura fria ${ }^{17}$. Embora os itens 14 e 15 tratem do controle de ruídos e da luminosidade, justifica-se a importância de um item sobre conforto no protocolo de cuidados, uma vez que o diagnóstico de câncer traz a consciência do risco de morte, que pode ser acompanhada de angústia e medo, influenciando, assim, o conforto do paciente ${ }^{18}$.

Excetuando-se os itens sobre dor oncológica e conforto, os demais foram construídos a partir das recomendações constantes do Clinical Practice Guidelines for the Management of Pain, Agitation, and Delirium ${ }^{6}$, que recomenda as melhores práticas para o manejo de dor, agitaçáo e delirium, a fim de melhorar os resultados clínicos de pacientes adultos internados em UTI. Portanto, vale destacar que o protocolo de cuidados foi desenvolvido com o propósito de contribuir para a uniformização de condutas assistenciais para pacientes críticos no contexto oncológico, de modo que todos os profissionais intensivistas possam observá-lo. Isto porque, no manejo e controle de outros fenômenos frequentes em UTI, programas de prevenção com agrupamento de recomendações ou combinação de boas práticas já apresentaram mais resultados positivos do que a realizaçáo de açôes ou recomendaçôes de forma isolada ${ }^{19}$.

\section{CONCLUSÃO}

Embora investigações futuras sejam necessárias para avaliar a aplicação prática e a viabilidade do uso do protocolo de cuidados construído, este apresentou adequadas validades de face e de conteúdo, mostrando-se, então, um instrumento promissor para o manejo do delirium em pacientes com câncer internados em UTI.

\section{CONTRIBUIÇÕES}

Bárbara Rocha Gouveia contribuiu na concepção e delineamento do estudo, análise de dados, interpretação dos resultados e redação do manuscrito. Rafael Tavares Jomar, Débora Cristina Leitão dos Santos e Tânia Cristina de Oliveira Valente contribuíram na revisão crítica e redação do artigo. Todos os autores aprovaram a versão final a ser publicada.

\section{DECLARAÇÃO DE CONFLITO DE INTERESSES}

Nada a declarar.

\section{FONTES DE FINANCIAMENTO}

Não há.

\section{REFERÊNCIAS}

1. Dessap AM, Roche-Campo F, Launay JM, et al. Delirium and circadian rhythm of melatonin during weaning from mechanical ventilation: an ancillary study of a weaning trial. Chest. 2015;148(5):1231-41. doi: https://doi. org/10.1378/chest.15-0525

2. Balas MC, Rice M, Chaperon C, et al. Management of delirium in critically ill older adults. Crit Care Nurse. 2012;32(4):15-26. doi: https://doi.org/10.4037/ ccn2012480

3. Mehta S, Cook D, Devlin JW, et al. Prevalence, risk factors, and outcomes of delirium in mechanically ventilated adults. Crit Care Med. 2015;43(3):557-66. doi: https://doi.org/10.1097/CCM.0000000000000727

4. Almeida ICT, Soares M, Bozza FA, et al. The impact of acute brain dysfunction in the outcomes of mechanically ventilated cancer patients. Plos One. 2014;9(1):e85332. doi: https://doi.org/10.1371/journal.pone.0085332

5. Gouveia BR, Jomar RT, Valente TCO. Delirium in cancer patients admitted to the intensive care unit: a retrospective study. Rev Bras Ter Intensiva. 2019;31(4):536-40. doi: https://doi.org/10.5935/0103507X.20190064

6. Barr J, Fraser GL, Puntillo K, et al. Clinical practice guidelines for the management of pain, agitation, and delirium in adult patients in the intensive care unit. Crit Care Med. 2013;41(1):263-306. doi: https://doi. org/10.1097/CCM.0b013e3182783b72

7. Kezar A, Maxey D. The Delphi technique: an untapped approach of participatory research. Int J Soc Res 
Methodol. 2016;19(2):143-60. doi: https://doi.org/10 $.1080 / 13645579.2014 .936737$

8. Alexandre NMC, Coluci MZO. Validade de conteúdo nos processos de construção e adaptação de instrumentos de medidas. Ciênc saúde coletiva. 2011;16(7):3061-8. doi: https://doi.org/10.1590/S1413-81232011000800006

9. Pasquali L. Princípios de elaboração de escalas psicológicas. Rev Psiquiatr Clin. 1998;25(5):206-13.

10. Payen JF, Bru O, Bosson JL, et al. Assessing pain in critically ill sedated patients by using a behavioral pain scale. Crit Care Med. 2001;29(12):2258-63. doi: https:// doi.org/10.1097/00003246-200112000-00004

11. Ely EW, Truman B, Shintani A, et al. Monitoring sedation status over time in ICU patients: reliability and validity of the Richmond Agitation-Sedation Scale (RASS). JAMA. 2003;289(22):2983-91. doi: https://doi. org/10.1001/jama.289.22.2983

12. Ely EW, Margolin R, Francis J, et al. Evaluation of delirium in critically ill patients: validation of the Confusion Assessment Method for the Intensive Care Unit (CAMICU). Crit Care Med. 2001;29(7):1370-9. doi: https:// doi.org/10.1097/00003246-200107000-00012

13. Trogrlić $Z$, van der Jagt M, Bakker J, et al. A systematic review of implementation strategies for assessment, prevention, and management of ICU delirium and their effect on clinical outcomes. Crit Care. 2015;19(1):157. doi: https://doi.org/10.1186/s13054-015-0886-9

14. Garcia TR. Classificação Internacional para a Prática de Enfermagem - CIPE ${ }^{\circledR}$. Versão 2017. Porto Alegre: Artmed; 2018.

15. Bastos AS, Beccaria LM, Silva DC, et al. Prevalence of delirium in intensive care patients and association with sedoanalgesia, severity and mortality. Rev Gaúcha Enferm. 2020;41:e20190068. doi: https://doi.org/10.1590/19831447.2020.20190068

16. Kolcaba KY. A taxonomic structure for the concept comfort. Image J Nurs Sch. 1991;23(4):237-40. doi: https://doi.org/10.1111/j.1547-5069.1991.tb00678.x

17. Vale CCSO, Líbero ACA. A espiritualidade que habita o CTI. Mental. 2017;11(21):321-38.

18. Gonçalves MCS, Brandão MAG, Duran ECM. Validation of the defining characteristics of the nursing diagnosis impaired comfort in oncology. Acta Paul Enferm. 2016;29(1):115-24. doi: https://doi. org/10.1590/1982-0194201600016

19. Vasconcelos JMB, Caliri MHL. Nursing actions before and after a protocol for preventing pressure injury in intensive care. Esc Anna Nery. 2017;21(1):e20170001. doi: https://doi.org/10.5935/1414-8145.20170001 